\title{
LOS SERVICIOS PÚBLICOS DE AGUA Y ENERGÍA EN LA PROVINCIA DE CORRIENTES: ASPECTOS COMPARADOS. ASI- METRÍAS Y TÉCNICAS REGULATORIAS EN LAS ÚLTIMAS CUATRO DÉCADAS
}

Miguel Andrés Goldfarb ${ }^{1}$

\section{Introducción}

Abordaremos un análisis comparado de las regulaciones existentes sobre dos servicios públicos esenciales en la Provincia de Corrientes. Nos referimos a las prestaciones de agua y energía eléctrica, pilares hoy en el concepto de calidad de vida y dignidad humana. Pretendemos describir las diferencias y analogías que existen en ambos campos, así como las posibles deficiencias regulatorias que aún hoy persisten a pesar de que desde 2007 existe un claro mandato constitucional sobre estas cuestiones. El análisis se circunscribirá a las últimas cuatro décadas — 1980 — cuando estos servicios pasaron a la órbita provincial.

A los fines del tratamiento del tema, en primer lugar haremos referencia a los fundamentos de la regulación y los antecedentes en la materia. Posteriormente abordaremos el impacto que la reforma de los años noventa y finalmente los aspectos normativos relacionados con dos elementos centrales en el análisis: los marcos regulatorios y la figura de los entes reguladores. Finalmente expondremos nuestras conclusiones.

${ }^{1}$ Abogado, Doctor en Derecho Público Política y Gobierno, Especialista en Derecho Administrativo y por la Facultad de Derecho y Ciencias Sociales y Políticas de la Universidad Nacional del Nordeste (U.N.N.E.); Especialista en Docencia Universitaria por la Universidad Nacional del Nordeste (U.N.N.E.); Master en Derecho Tributario (Universidad de Barcelona, España U.B.) y Especialista en Asesoría Jurídica de Empresas (Facultad de Derecho de la Universidad de Buenos Aires UBA); Docente por concurso - categorizado investigador- de las asignaturas Derecho Financiero y Tributario y Derecho Administrativo I de la carrera de Abogacía de la Facultad de Derecho de la U.N.N.E; Docente de posgrado. Delegado en Corrientes del Cuerpo de Abogados del Estado Nacional (Procuración del Tesoro de la Nación P.T.N.) Autor y coautor de numerosos artículos y libros sobre temas de derecho público. 


\section{Los fundamentos regulatorios y los antecedentes en ambos casos-agua y energía- en Corrientes}

Sabido es que el sistema definido originariamente por Adam Smith, en virtud del cual, a través de una mano invisible los intereses de productores y consumidores convergen necesariamente de modo tal que se logra una posición de equilibrio y bienestar general no es perfecto ni se da en la realidad de modo ideal. Existen pues, las denominadas "fallas de mercado" en donde la máxima e ideal relación resultado-recurso no es posible y el libre juego del mercado no lo resuelve.

Se ha sostenido que en las denominadas fallas del mercado, los demandantes no satisfacen sus necesidades adecuadamente y aún dispuestos a pagar un precio más alto, tampoco su necesidad será resuelta, encontrándonos ante un caso de ineficiencia económica que la acción del Estado debería subsanar. ${ }^{2}$ Entre las grandes fallas de mercado encontramos: a) bienes públicos b) contaminación ambiental y c) monopolios naturales.

Claramente los servicios de de agua y energía categorizan dentro de los monopolios naturales. Un mercado monopólico es aquel que por determinadas circunstancias se limita a tener un solo oferente o bien existe uno que detenta una posición de dominio en ese mercado. El monopolio es considerado disvalioso desde el momento en el que facilita el abuso del actor fuerte del mercado contra los intereses de los usuarios y consumidores, e incluso contra potenciales consumidores y competidores.

Una empresa monopólica maximiza al igualar el ingreso marginal con su costo marginal, siendo el precio mayor que el costo medio. Opera en eficiencia $\mathrm{x}$ (para él) pero no en eficiencia del sistema, puesto que al no haber otros oferentes, no opera el costo medio mínimo. ${ }^{3}$

${ }^{2}$ Scandizo, Daniel. Contribuciones a la gestión pública. Universidad Católica de Córdoba. Serie PROFIM. Editorial El copista. Córdoba. Argentina. 2006, p. 33.

${ }^{3}$ En términos puramente económicos, el monopolio es denominado "natural" cuando se caracteriza porque al alcanzar un elevado volumen de producción la incidencia de los costos fijos se reduce respecto de cada unidad adicional, reduciéndose el costo medio y produciéndose economía a escala. Es el caso de la llamada "economía a escala". El costo marginal es menor que el costo medio promedio; es decir que alcanzando cierto volumen de producción (economía a escala) los costos disminuyen. Consecuentemente el mercado es más eficiente en la medida en la que cuenta con un solo oferente. Consecuentemente, esta especie de monopolios, a diferencia de los otros, opera en el campo de los costos decrecientes. Dos empresas operan con costos medios mayores que una. 
Recuerda Macon que Estados Unidos dictó a fines del siglo IXX la ley Sherman anti-trust, a partir de los cuales se resolvieron varios casos: standar oil, Rockefeller y ATT. Este autor explica también que la motivación de las normas antimonopólicas no son sólo de naturaleza económica, sino también políticas: el Estado busca evitar que se rompa el equilibrio social y de poder, el cual es fundamental para el sistema democrático. ${ }^{4}$

En general, los servicios públicos constituyen monopolios naturales, asumiendo costos de inversión de capital altos y costos hundidos.

En su caso, la doctrina ha señalado que existen una serie de típicas características que son propias del monopolio natural:

a) Alta inversión inicial del capital.

b) Prestación de servicios o bienes valorados como esenciales (p.e. agua potable).

c) Imposibilidad de almacenamiento en casos de baja demanda.

d) Necesidad de una conexión en red.

e) Mercado atractivo para obtener rentas. ${ }^{5}$

En un sentido más amplio se considera monopolio natural también a aquel en donde la producción de una sola empresa representa costos menores que la producción de varias empresas por separado.

Los marcos regulatorios que el Estado debe propiciar en los casos de monopolios naturales implican un doble desafio, en razón de que además de proteger a los usuarios de prácticas abusivas en materia de tarifas, calidad y cantidad del servicio ofrecido, también debe tutelar a la empresa prestataria, evitando la entrada de competidores que desmembren el mercado, tornándolo antieconómico. ${ }^{6}$

${ }^{4}$ Macon, Jorge. Economia del Sector Público. Ed. Mc Graw Hill Interamericana. Bogotá. Colombia. 2002, p. 48.

${ }^{5}$ Bustamante, Jorge Eduardo. Desregulación. Entre el Derecho y la Economía. Abeledo Perrot. Buenos Aires. 1993, p. 107.

${ }^{6}$ Cabe recordar que la situación de monopolio natural, a menudo, ha sido tratada con tres posibles soluciones:

Empresas públicas, en donde ante una situación deficitaria es asumida por los contribuyentes.

Empresas privadas subsidiadas, en cuyo caso, al existir situación de déficit es soportada por las cuentas fiscales.

Empresas privadas reguladas por el Estado sin régimen de subsidios. En este caso, la situación posible de déficit no es afrontada por el gasto público. 
Es apropiado regular un mercado que funciona bajo la modalidad de monopolio natural a los efectos de que éste subsista, a la inversa de los monopolios artificiales, en los que se regula a los efectos de facilitar la entrada de competidores y favorecer así la competencia. Como hemos visto anteriormente, la regulación busca que no se desmembre el mercado - protege al productor pero también se tutelan los intereses de los usuarios y consumidores; más aún cuando estamos en presencia de un bien esencial o preferente.

Jorge Eduardo Bustamante (1993) ha sostenido que se han desarrollado diversas teorías a los efectos de justificar la regulación estatal.

En primer lugar, se desarrolló teoría del interés público, que a su vez ha sido la más aceptada, y que encuentra como fundamento de la regulación de un sector de la economía la necesidad de que el Estado asegure el "bien común", corrigiendo las ineficiencias del mercado. Tal sería el caso del servicio de agua y saneamiento, de indudable impacto social y con estrecha relación en desarrollo humano que el Estado debe tutelar como garante del interés público.

En el caso de los servicios públicos el Estado ha tomado dos caminos: a) creado empresas públicas u b) otorgado la gestión y organización del servicio a una empresa privada; siempre a través de la técnica jurídico administrativa de la concesión. En párrafos subsiguientes veremos de qué modo Corrientes afrontó la disyuntiva recurriendo, precisamente a la forma de empresa pública y luego a la concesión.

Siempre siguiendo a Bustamente, encontramos la teoría del acuerdo colectivo, en razón de la cual, la intervención estatal halla fundamento en el famoso "dilema del prisionero", donde se permitiría desbloquear los dilemas de acción colectiva (juego de conveniencias) a través de las regulaciones que suplantan la voluntad de todos los actores, incluso la de aquellos que se oponen a un acuerdo por motivos estratégicos, aun sabiendo de su conveniencia (free riding). De este modo, la regulación estatal vendría a forzar un acuerdo colectivo o social.

Bustamante critica esta última teoría, pues considera que desvirtúa la dinámica del libre consenso y postula la tesis de los costos de transacción, según la cual la verdadera reforma institucional consiste en eliminar o reducir los costos de transacción.

Constituyen costos de transacción - en sentido muy amplio- aquellos aspectos institucionales y fácticos, así como todas las circunstancias que difi-

\footnotetext{
${ }^{7}$ Bustamante, Jorge Eduardo, ob. cit., p. 37 y siguientes.
} 
cultan llegar a un acuerdo sobre un interés en común. En este orden de ideas, las regulaciones estatales constituyen costos de transacción. ${ }^{8}$

Desde esta óptica, el fundamento de la regulación esta dado, no por la creación de restricciones al mercado, sino por la creación de instituciones que tienden a fortalecerlo; tales como han sido para la economía el dinero, la letra de cambio o la responsabilidad limitada. Para este autor, serán las instituciones que impulsen la competencia aquellas que eliminen las fallas del mercado, y no las tradicionales restricciones regulatorias.

Finalmente, encontramos la denominada teoría económica de las regulaciones, partiendo de dos premisas: a) toda regulación tiene costos; b) la regulación puede explicarse desde la ley de la oferta y la demanda.

En efecto, desde esta concepción el Estado es demandado por regulaciones, pues mejora así la rentabilidad de los sectores beneficiados, siendo esto consecuencia de la natural búsqueda de la maximización.

Habrá que ver entonces en cada caso, quienes o cuales son en cada caso, los realmente favorecidos por las regulaciones de los distintos sectores de la economía y del sector productivo del país.

De todos modos, la razón de ser de El caso del "mercado" de agua potable y saneamiento posee características notables atento la naturaleza esencial que reviste el bien del que se trata, tal como es definido en nuestros días el acceso al agua saludable como derecho humano. Es por ello que postuló como fundamento de la intervención administrativa la existencia de un interés público concreto y perfectamente definido, como lo es, asegurar la calidad y eficiencia de la prestación de un servicio que cubre una necesidad esencial y vital para la condición humana.

Del mismo modo, la tendencia actual es reconocer que el acceso al servicio eléctrico también constituye hoy un derecho elemental, de modo tal que la jurisprudencia, incluso aquella emanada de Tribunales de Corrientes, ha establecido franquicias del $100 \%$ en el pago de la tarifa ante determinados supuestos extremos. ${ }^{9}$

${ }^{8}$ Ídem, p. 40 y siguientes.

${ }^{9}$ Causa: Meza Marta c/ Dirección Provincial de Energía Eléctrica y Estado de la Pcia. de Corrientes s/Amparo Expte. 40180/9. Superior Tribunal de Justicia. [www.juscorrientes.gov.ar]. 
El objeto de la intervención del Estado es, de una manera o de otra, la tutela del bien común, elemento que nos conduce a la noción de bienestar general y resguardo del interés público. Tales expresiones tienen su fundamento constitucional en el propio artículo 75 inciso $18 \mathrm{y}$ en el concepto de poder de policía ya consagrado en el artículo 14 del texto Constitucional.

Dicho lo anterior, cabe indicar que el supuesto de las prestaciones de agua potable y energía eléctrica constituye a todas luces un claro ejemplo de monopolio natural y que, en el caso de Corrientes, como en la generalidad, ha sido abordado través de la creación de empresas públicas, una de ellas luego concesionada.

Si bien en un principio la provisión de los servicios se hacía a través de prestadores privados, luego, bajo el paradigma del estado de bienestar y una visión intervencionista del Estado desde los inicios del siglo pasado, los servicios estuvieron a cargo de empresas públicas en diferentes formatos.

El 10 de setiembre de 1980, a través de una Resolución emanada conjuntamente de los Ministerios de Economía-Interior, y por aplicación de la Ley Nacional $\mathrm{N}^{\circ} 18586$, se dispuso la devolución de los servicios a las provincias, lo que se concretó mediante un Convenio ratificado por la antes citada Ley Provincial No 3558/80. La vieja e histórica "Agua y Energía" que desde 1972 funcionaba en la órbita Nacional volvió así a la esfera provincial.

Fue así que al operarse la mencionada transferencia, se dictaron en Corrientes sendos Decretos Leyes $\mathrm{N}^{\circ} 3573$ y $\mathrm{N}^{\circ} 3588$ de creación de las respectivas empresas públicas (entidades descentralizadas desde el punto de vista de la organización administrativa) que tomaron a su cargo la prestación de Agua y Electricidad.

A través de la Ley (en realidad Decreto ley) N³573 se instituyó la "Administración de Obras Sanitarias" que tomó a su cargo la prestación del servicio de agua potable y cloacas. Se fijaron sus competencias y composición, estableciéndose que el organismo tomaba a su cargo la prestación y las actividades vinculadas al servicio público de agua potable y saneamiento. ${ }^{10}$

${ }^{10} \mathrm{El}$ artículo primero de la Ley $\mathrm{N}^{\circ} 3573$ establece: créase como ente autárquico con domicilio en la ciudad de Corrientes, donde tendrá asiento su sede central, la Administración de Obras Sanitarias de la Provincia de Corrientes (AOSC), con personalidad jurídica de derecho público y de derecho privado. El Ente gozará de autarquía financiera y administrativa. Art. $2^{\circ}$ : La Administración de Obras Sanitarias de la Provincia de Corrientes tiene como finalidad consolidar, expandir y fomentar los servicios sanitarios y tendrá a su cargo el estudio, proyecto, construcción, 
En materia eléctrica, de modo paralelo al caso del agua y saneamiento, el Gobierno de la Provincia de Corrientes creó la Dirección Provincial de Energía (D.P.E.C.), reconociéndole la condición ente autárquico, con dependencia funcional de la Secretaría de Estado de Obras y Servicios Públicos (actualmente Subsecretaría de Energía).

De conformidad con lo normado en el art. 3 de la Ley $\mathrm{N}^{\circ} 3588$, su objeto es la promoción, estudio, proyecto, ejecución y/o explotación de obras y servicios de aprovechamiento energético, así como la generación, transporte, distribución y comercialización de energía eléctrica, y la coordinación de los mismos servicios prestados por los terceros.

renovación, ampliación y explotación de las obras de provisión de agua y saneamiento urbano, la exploración, alumbramiento y utilización de las aguas subterráneas en la ciudad Capital y ciudades y pueblos de la Provincia. Asimismo, entre las principales competencias la ley determinó: Art. $4^{\circ}$. Para el cumplimiento de los fines y objeto establecidos en el art. $2^{\circ}$, el Ente tendrá las siguientes atribuciones: a) Administrar los bienes de su patrimonio. Realizar operaciones de créditos con instituciones oficiales, privadas o mixtas, nacionales o extranjeras, previa intervención por el conducto pertinente, del Ministerio de Economía y autorización del Poder Ejecutivo; b) Otorgar poderes generales o especiales y revocarlos, actuar en juicio como demandante o querellante y demandada y celebrar arreglos judiciales; c) Celebrar toda clase de contratos vinculados a su objeto con excepción de lo atingente a contratación de personal; d) Efectuar sus contrataciones según las normas de la presente ley, de reglamentación que se dicte, de la ley general de obras públicas y de la ley de contabilidad, que será de aplicación supletoria esta última, en lo que no resulte modificada por la presente. Posteriormente encontramos los artículos 30 y 31 de la misma ley, que expresamente ponen a cargo del Estado el servicio, que a su vez luego es definido como público. Dispone el artículo 30 que: "La Administración de Obras Sanitarias de la Provincia de Corrientes prestará los servicios de agua corriente, desagües cloacales y aquellos otros que preste en lo sucesivo, conforme al reglamento que dicte"; y seguidamente reza el artículo 31 : "Todo inmueble comprendido en las zonas dotadas del servicio público estará obligado al uso y su propietario al pago del mismo, cuando el inmueble sea habitable; y cuando en el mismo no se encontrase de ninguna índole no podrá exigirse pago alguno" (Texto del artículo según Ley $\left.N^{o} 5502\right)$. Asimismo, de modo concordante el artículo 45 del mismo texto legal menciona los términos "servicios públicos" estableciendo: "Declárese de utilidad pública y sujetos a expropiación o constitución de servidumbres, los inmuebles, muebles, instalaciones, derechos y bienes en general, cualquiera sea su naturaleza jurídica, que fueren necesarios para el cumplimiento de los planes de trabajo correspondientes a la prestación de los servicios públicos a cargo del Organismo, quien determinará en cada caso, los bienes que serán sometidos a expropiación o constitución de servidumbres". Si bien no existe en Corrientes una ley marco regulatoria del servicio, tal como sí sucede en el caso de las Provincias de Chaco y Misiones, o como en el plano nacional las Leyes $\mathrm{N}^{\circ} 24065$ y $\mathrm{N}^{\circ} 24076$ relativas al servicio de electricidad y gas, entiendo que de la inteligencia de los artículos transcritos en el párrafo precedente, surge claramente la publicatio del servicio de prestación de agua potable en Corrientes. Recordemos que ésta puede ser expresa o tácita, siempre y cuando emane de una declaración de naturaleza legislativa, toda vez que implica una especie de "captura" de una parte del mercado. 
El segundo párrafo del citado art. 3 convierte a la Dirección Provincial de Energía en la ejecutora de las decisiones en materia energética, cuya competencia pertenecía a la entonces Secretaría de Estado de Obras y Servicios Públicos.

Este fue el escenario regulatorio hasta la Reforma del Estado que se inició en 1989. Hasta aquel momento, la Provincia prosiguió con un esquema tradicional de empresa pública bajo la forma de ente autárquico descentralizado sin la existencia aún de marcos regulatorios ni entes reguladores. La posición del usuario no tenía raigambre constitucional y las fuertes transformaciones de los mercados estaban recién por iniciarse.

Ciertos institutos, como los marcos regulatorios, los entes reguladores y la normativa relativa al usuario o consumidor recién aparecieron en nuestro mapa jurídico en el plano nacional en los años noventa, cual claros ejemplos de instituciones que caracterizaron al disímil y cuestionado proceso de "privatización" de los servicios públicos.

En párrafos siguientes veremos de qué modo la impronta del Estado mínimo repercutió asimétricamente en la Provincia de Corrientes. Ello, sin perjuicio de que esta Provincia en materia de agua y cloacas tuvo el extraño privilegio de ser vanguardia. No en materia de calidad regulatoria pero sí dado lo rápido del proceso de transferencia del servicio a manos privadas.

\section{La impronta de las reformas de los años 90: el inicio de las asimetrías regulatorias}

Iniciado el proceso de reformas de 1989, la Provincia de Corrientes adhirió a la Ley Nacional $N^{\circ} 23696$ a través de la Ley $N^{\circ} 4366$, publicada en el Boletín Oficial el cinco de octubre de 1989.

De modo casi inmediato, el servicio de agua potable y saneamiento fue concesionado, no ocurriendo lo mismo con el sector eléctrico que vivió escenarios diferentes a los cuales nos referiremos infra. ${ }^{11}$

En materia de agua potable de modo análogo y paralelo a lo sucedido con el Estado Nacional durante los años noventa, ${ }^{12}$ en 1991, la Provincia de Corrientes concesionó el servicio.

En primer término, a través del Decreto 5120/90 llamó a Licitación Pública Nacional e Internacional para proceder a otorgar la concesión integral de explotación de los servicios de agua potable y desagües cloacales que, desde

${ }^{11}$ Falta nota 
1981 venían siendo prestados por Obras Sanitarias de la Provincia (A.O.S.C.) en las principales localidades de Corrientes. ${ }^{13}$

Mediante el Decreto 1161/91 se adjudicó la concesión a la empresa Aguas de Corrientes S.A. - en ese momento S.A. en formación, compuesta por diversos grupos empresarios-. Fue la Ley $\mathrm{N}^{\circ} 4366$ de adhesión a la Ley $\mathrm{N}^{\circ}$ 23696 (Reforma del Estado Nacional) el instrumento legislativo que operó como base para la realización del procedimiento privatizador. Se buscaba la mayor eficiencia en la prestación integral del servicio. Recordemos que la Ley $\mathrm{N}^{\circ} 23696$ constituyó un verdadero estatuto de las privatizaciones de aquella década. La ley continúa vigente.

Luego se dictó otro Decreto (4467/91) por medio del cual se aprobó el modelo de Acta de Transferencia a los fines de la toma de posesión de la "Empresa Aguas de Corrientes S.A.".

La administración encuadró jurídicamente la relación con los prestadores a través de la clásica figura contractual del Derecho Administrativo: la concesión de servicio público. La explotación es a costa y riesgo del concesionario bajo la vigilancia y control del ente concedente. El plazo original de la Concesión que abarcaba a los principales Departamentos y a la Capital Provincial, era de treinta años, extendido luego en cinco más a partir de la renegociación aprobada en 2004. Si bien hubo distintos ajustes contractuales ya desde el mismo inicio de la relación jurídica, la renegociación más relevante tuvo lugar partir de la crisis nacional de los años 2001-2002 que asoló al país.

En el crítico contexto del año 2002 la Legislatura de la Provincia de Corrientes adhirió a la Ley $\mathrm{N}^{\circ} 25561$ a través de la Ley $\mathrm{N}^{\circ} 5429$ de fecha 15 de mayo de aquel año(B.O. 21/05/2002), facultando al P.E. a renegociar los contratos de servicios públicos afectados por la declaración de emergencia efectuada.

Se inició así un proceso de renegociación que tuvo como característica sobresaliente que no activó mecanismos de participación ciudadana — por ejemplo audiencias públicas o consultas a asociaciones de usuarios y consumidores- ni contó con la intervención de organismos públicos o privados vinculados al servicio, tal como sí sucedió en el plano nacional con la creación de la Unidad de Renegociación y Análisis de los Contratos de Servicios Públicos dispuesta por Decreto 311/03 y que funciona en la órbita de los Ministerios de Planificación

${ }^{12}$ Debería aquí recordarse el inicio del proceso de privatizaciones en el plano nacional a partir de la Ley de Reforma del Estado $N^{\circ} 23696$.

${ }^{13}$ Corrientes, Goya, Curuzú Cuatiá, Mercedes, Santo Tomé, Paso de los Libres, Monte Caseros, Esquina, Bella Vista y Saladas. 


\section{y Economía.}

Como corolario del proceso de renegociación se aprobó un marco acuerdo a través del Decreto 2962 /2004 en donde los principales aspectos fueron:

- Continuidad de la empresa al frente del servicio en "estricto cumplimiento de los requerimientos de calidad previstos en el contrato de concesión" (Cláusula primera)

- Diferimiento de la puesta en funcionamiento de las plantas de tratamiento pactadas para Corrientes y Goya para agosto de 2010

- Prórroga del contrato por el término de cinco años.

- Se dejó sin efecto el derecho de cobro sobre sitios baldíos (un servicio inexistente, claro) y se estableció un régimen especial para propiedades afectadas al régimen de propiedad horizontal que no fueran consorcios. Se estipuló la aplicación de un importe mínimo mensual equivalente a $20 \mathrm{~m} 3$ por unidad funcional (Cláusulas quinta y octava).

- Desistimiento del derecho y de las acciones judiciales interpuestas por la empresa contra el Estado en virtud del dictado del Decreto 1238/00 que había declarado nulos los Decretos 4289 y 4293 del año 1997 y el No $3132 / 98 . .^{14}$

- Se acordó la validez y vigencia de la conciliación y compensación de créditos y deudas detalladas en el Decreto $4293 / 97$ y convenio de fecha 27 de octubre de aquel año.

- Se fijaron nuevos cargos tarifarios que no fueron homologados por el Poder Ejecutivo.

- Ampliación de las localidades servidas por la empresa (Empedrado,

${ }^{14}$ A través de los Decretos señalados de los años 1997 y 1998, la Provincia de Corrientes autorizó al ente regulador a celebrar un convenio con la concesionaria modificando el régimen tarifario. Se crearon dos cuadros tarifarios: uno normal y otro de tipo social para un máximo del $7 \%$ de los clientes. Se dispuso un complejo sistema de condonaciones a los morosos asumiendo la Provincia parte de la deuda de los beneficiarios de la tarifa social hasta diciembre de 1996 (un tercio). Asimismo la deuda a cargo del Estado fue declarada sujeta a compensación con créditos del Estado central o descentralizado con relación a la concesionaria, "o mediante la mecánica o procedimientos de cancelación que con efectos análogos se estableciera de conformidad al régimen cuya implementación se le encomendó al Ministerio de Obras Públicas". El segundo Decreto (4293/97) aprobó un modelo de convenio de conciliación y compensación de créditos y deudas recíprocas. Finalmente el tercer decreto anulado ponía a cargo del Estado una deuda con el BID que de conformidad con el contrato de concesión se encontraba en cabeza de la empresa prestataria. La Intervención Federal dictó el Decreto 1238/00 declarando nulos los decretos citados e inexistentes los acuerdos suscriptos en consecuencia, considerando asimismo que tales actos administrativos implicaban una modificación sustancial y arbitraria a lo establecido en el contrato de concesion y los pliegos de bases y condiciones. 
Yapeyú y Santa Lucía).

- Se pactó que con relación a las prestaciones de agua y cloaca mediante el mecanismo de macromedición a núcleos habitacionales fuera del área de concesión, que la concesionaria procedería en el término de seis meses a facturar de forma individual y a cada usuario el precio de tales prestaciones.

- Puesta en funcionamiento de la comisión mixta (con dos integrantes por parte) con el objeto de continuar las negociaciones y efectuar una revisión integral del contrato de concesión en la búsqueda de condiciones que tornen posible la continuidad, regularidad y eficiencia del servicio en un marco de cooperación entre ambas partes (Cláusulas decimocuarta a decimoctava).

Volveremos luego sobre algunas deficiencias en materia regularoria en esta concesión caracterizada primordialmente por un la falta de un verdadero ente regulador y la inexistencia de una Ley de Marco Regulatorio.

En el contexto sociopolítico señalado, en materia eléctrica, se dictó en el plano nacional el marco regulatorio del servicio público de transporte y distribución de energía, sancionándose así la Ley $\mathrm{N}^{\circ} 24065$. Se creó también el ente regulador de la energía ENRE.

La citada norma estableció que el sector eléctrico se articula en tres segmentos independientes, a saber: generación, transporte y distribución. ${ }^{15}$

A partir de lo prescripto en el citado artículo podemos extraer dos definiciones muy importantes: a) se produjo una desintegración vertical del sistema que permite desconcentrar en tres etapas el servicio, evitando así un auténtico monopolio que reúna en una sola empresa todo el proceso eléctrico; y b) caracterizar al transporte y distribución como servicios públicos, dotando entonces a tales actividades de las condiciones legales de continuidad, regularidad, generalidad y obligatoriedad.

Si bien operó la transferencia al sector privado en el ámbito nacional, no sucedió lo mismo en la Provincia de Corrientes. Comenzó allí una especie de

${ }^{15}$ Dice el artículo primero de la Ley $N^{\circ}$ 24065: Caracterízase como servicio público al transporte y distribución de electricidad. Exceptúase, no obstante su naturaleza monopólica, el régimen de ampliación del transporte que no tenga como objetivo principal la mejora o el mantenimiento de la confiabilidad que, en tanto comparta las reglas propias del mercado, será de libre iniciativa y a propio riesgo de quien la ejecute. La actividad de generación, en cualquiera de sus modalidades, destinada total o parcialmente a abastecer de energía a un servicio público será considerada de interés general, afectada a dicho servicio y encuadrada en las normas legales y reglamentarias que aseguren el normal funcionamiento del mismo. 
bifurcación de rumbos entre ambos servicios. No es que los sucesivos gobiernos provinciales no hayan intentado concesionar también el servicio eléctrico, pero dadas las dificultades políticas de aquellos años, el traspaso al sector privado no prosperó. ${ }^{16}$

Concluida la intervención Federal 1991-1993, en el año 1995, imbuida de la concepción ideológica vigente en aquellos momentos que propiciaba el Estado subsidiario, se dictó la Ley $\mathrm{N}^{\circ} 4921$, en virtud de la cual básicamente se dispuso la autorización para que el Poder Ejecutivo procediera a "privatizar" la Dirección Provincial de Energía Eléctrica. Entre los puntos sobresalientes se encuentran: a) autorización para privatizar total o parcialmente. En el caso de privatización parcial, el Estado no podía reservarse más del 49\% de las acciones; b) la transformación de la D.P.E.C. en Sociedad Anónima, conforme a la Ley $\mathrm{N}^{\circ} 19550$ y sus modificatorias; ${ }^{17}$ y c) La creación de una comisión bicameral de seguimiento.

Los continuos vaivenes políticos sucedidos en la Provincia impidieron que se operara la concesión del servicio, con lo cual este prosiguió a cargo del Estado a través de la Dirección Provincial de Energía Eléctrica regida por la

16 "Los conflictos generados a partir del bloqueo del Colegio Electoral impidieron por desde el año 1991 y hasta el año 1993 la formación de un gobierno democráticamente elegido. Durante este periodo tres interventores federales administraron la provincia. Esta situación probablemente haya truncado el proceso de trasferencias de las empresas del Estado de la Provincia al sector privado. En efecto en el año 1991 se realizaron estudios sobre la factibilidad de la privatización de la Dirección Provincial de Energía. El avance más significativo hacia la privatización luego de las intervenciones federales durante el gobierno de Raúl Rolando Romero Feris con el dictado de la Ley N $\mathrm{N}^{\circ} 4921$ del año 1995. El quiebre del Pacto Autonomista Liberal a partir de la renuncia del Vice Gobernador y de varios ministros y conformación de una nueva fuerza política, el PANU, dirigida por el gobernador en ejercicio, pudo haber sido una de las variables que influyeron en la no aplicación de esta ley. Finalmente, la ley fue derogada en el año 2007, con el dictado de la Ley $\mathrm{N}^{\circ} 5799$, en un contexto nacional diferente. En el año 1999 con el dictado de la Ley $\mathrm{N}^{\circ} 5378$ durante el gobierno de coalición que sustituyó al gobierno elegido del Partido Nuevo, se realizó un nuevo avance en post de la privatización del servicio. Esta medida estaba ligada a la obtención de un préstamo de salvataje por parte del gobierno nacional para la provincia de Corrientes, incluía además la privatización del Banco de Corrientes que había sido estatalizado por Raúl Rolando Romero Feris. Ante una marcada situación de ingobernabilidad la intervención federal dispuesta por el gobierno de la Alianza dejó sin efecto la privatización de la DPEC. El gobierno de Arturo Colombi, otorgó el gerenciamiento de la empresa al mismo grupo que administra el servicio de agua y saneamiento, pero la experiencia de la gestión privada de algunos aspectos de la empresa de energía no fue positiva. El último gobierno Ricardo Colombi impulsó un nuevo régimen del servicio eléctrico (que será analizado) la conformación de una empresa privada para la explotación de energías alternativas" (Ver Medina, Marcos. IX Congreso Nacional de Derecho Político. Rosario, Junio de 2012. Ponencia presentada en la Comisión Estado y Regulación). 
vieja Ley $\mathrm{N}^{\circ} 3588$.

Posteriormente, se dictó la Ley $\mathrm{N}^{\circ} 5378$; un verdadero marco regulatorio ${ }^{18}$ que estableció expresamente la condición de servicio público de la distribución de la energía eléctrica (artículo primero). Asimismo esta ley determinó —en su artículo sexto- que:

Las expresiones "servicio público"y "distribución" significan la distribución regular y continua de energía eléctrica para atender las necesidades indispensables y generales de electricidad de los usuarios de una colectividad o grupo social determinado que no tengan la posibilidad de contratar su abastecimiento en forma independiente.

Esta norma no se puso en práctica y fue luego derogada.

A los fines de la transformación de la empresa pública en una sociedad anónima el Poder Ejecutivo dictó el Decreto 2986/95, en razón del cual se dispuso la creación de la empresa de distribución de energía "Taragüi Sociedad Anónima", conforme lo establecido en la Ley $\mathrm{N}^{\circ} 19550$. Estos instrumentos normativos tendían a materializar la privatización, que finalmente no operó en la realidad hasta que se dictó la Ley $\mathrm{N}^{\circ} 5799$.

El artículo 64 de dicha Ley dispuso la creación del ente regulador, que fue luego instrumentado a través del Decreto 2988/95, pero que nunca funcionó.

${ }^{17}$ El artículo tercero de la Ley N 4921 establecía: "El Poder Ejecutivo Provincial determinará las bases y condiciones de la privatización y el llamado a licitación que deberá tener en cuenta la siguiente: a) La transformación de la D.P.E.C. en Sociedad Anónima, conforme a la Ley $\mathrm{N}^{\circ} 19550$ y sus modificatorias. b) Valuación del patrimonio de la empresa y su precio de venta por entidades públicas y/o privadas que aseguren su objetividad, en el precio base a fijarse. Forma y condiciones de pago. c) Requisitos técnicos, económicos y financieros de o las empresas que serán responsables del servicio. d) Fijación del método de determinación del cuadro tarifarlo, por el tiempo de concesión de los servicios; tendientes a su baja en el tiempo. e) Determinación de la concesión del servicio público conforme la legislación vigente, por un plazo no mayor de treinta (30) años y respetando los servicios que presten las cooperativas eléctricas existentes. f) Prever un sistema de participación en el capital para el personal mediante la venta de acciones. g) Establecer las metas y estándares que deberá alcanzar la calidad del servicio a prestar por la empresa. h) Creación o determinación del Ente Regulador encargado de ejercer como autoridad de aplicación, el marco regulatorio del servicio y sus tarifas y políticas energéticas".

${ }^{18}$ Determinó la condición de servicio público, el rol del Estado, la necesidad de licitación, pago de cánones, los objetivos, la regulación de las tarifas, la definición de conceptos tales como usuarios y grandes usuarios, las servidumbres administrativas, lo atinente a los derechos y obligaciones de las partes, facultades de la administración, la creación de un ente regulador, derechos de los usuarios, etc. 
En este contexto de reformas privatizadoras, propias de la década de los años noventa, encontramos que de la lectura y análisis de los aspectos jurídicos señalados y de los hechos descriptos, queda clara la intención de dotarle al Estado de un rol subsidiario por parte del Estado que, sin desaparecer, se limitó a fijar estándares mínimos y deja a las fuerzas del mercado la atención de estas necesidades primarias.

Desde el año 1995 hasta la actualidad se continuó prestando el servicio de distribución eléctrica a través de la Dirección Provincial de Energía de Corrientes (DPEC) y desde 1991 el servicio de agua potable continua en manos de una empresa concesionaria.

En el siguiente punto analizaremos dos de los aspectos más relevantes que condicionan negativamente la regulación de ambos servicios: la normativa constitutiva de los marcos regulatorios y la creación de los denominados "entes reguladores". 